The Journal of Animal \& Plant Sciences, 30(3): 2020, Page: 649-654

ISSN (print): 1018-7081; ISSN (online): 2309-8694

\title{
PHOTOMETRY-BASED PREDICTION MODEL FOR THE CONTENT OF OLEANOLIC ACID IN PERIPLOCAFORRESTIISCHLTR.
}

\author{
Y. Long ${ }^{1,2^{*}}, \mathrm{X} . \mathrm{Yi}^{2}$ and W. Yang ${ }^{1 *}$ \\ ${ }^{1}$ College of Pharmacy, Guizhou University of Traditional Chinese Medicine, 50 Shidong Road, Guiyang, 550001, \\ Guizhou, P. R. China \\ ${ }^{2}$ Second Affiliated Hospital, Guizhou University of Traditional Chinese Medicine, 83 Feishan Street, Guiyang, 550002, \\ Guizhou, P. R. China \\ *Co-corresponding Author’s email: 293398231@qq.com; ywd_680708@sina.com
}

\begin{abstract}
To realize rapid, low-cost identification and ensure quality control of Periplocaforrestii Schltr., a Miao ethnomedicine, a prediction model for oleanolic acid was built based on photometry. The total saponin content and oleanolic acid content inPeriplocaforrestiiSchltr. were analyzed by spectrophotometry and HPLC, respectively, and good linearity was observed for both of these methods. The Pearson correlation coefficient of the two data groups was 0.99 , indicating a significant positive correlation. Thus, a prediction curve for the content of oleanolic acid in PeriplocaforrestiiSchltr. was constructed by fitting the relationship between these two indicators to a parabolic curve $\left(\mathrm{R}^{2}=0.987\right)$. This model was successfully applied to predict the content of oleanolic acid in two samples, with a discrepancy rate of $<3 \%$ between of the predicted values and the experimental values. This method exhibited an excellent goodness of fit and showed high precision for determining the content of oleanolic acid in Periplocaforrestii Schltr. samples. As the total saponin content can be determined using simple photometric methods, this prediction model allows rapid and convenient determination of oleanolic acid inPeriplocaforrestiiSchltr. This approach may be useful for identifying natural medicines in ethnic areas.
\end{abstract}

Keywords: Periplocaforrestii Schltr.; Photometry; Saponin; Oleanolic acid; Content prediction; Quality control. https://doi.org/10.36899/JAPS.2020.3.0077 Published online March 25, 2020

\section{INTRODUCTION}

Periplocaforrestii Schltr., a plant in the Periploca L. family of Asclepiadaceae, is widely distributed in places such as Guangxi, Yunnan, Guizhou, and Tibet (Chen et al., 2017). This species has been identified as an ethnomedicine with obvious immunosuppressive activity and is used in the folk treatment of closed soft-tissue injuries and diseases such as rheumatism and rheumatoid arthritis (Hu et al., 2007; Zhang et al., 2011; Liang et al., 2015). Other reported effects include dispelling wind, removing dampness, relieving stagnation, killing pain, promoting blood circulation, and removing meridian obstructions (Chen et al., 2017). Multiple scholars have investigated the pharmacological effects of Periplocaforrestii Schltr. (Chen et al., 2005; Lu et al., 2011), and studies on its chemical composition have also achieved substantial results (Liang et al., 2017; Xu et al., 2011; Zhao et al., 2014). In addition, drug developers and researchers have focused on the quality control of this remarkable and resource-rich ethnomedicine (Zhao et al., 2016; Xu et al., 2016; Li et al., 2015). To date, these studies have mainly relied on various high-precision chromatographic and mass spectrometric detection methods. However, these methods generally have strict environmental requirements, high costs, and complicated operating procedures, which are a heavy burden for economically underdeveloped ethnic areas and extremely unfavorable for the promotion of quality control of local drugs. To solve this problem, it is necessary to develop a low-cost and rapid quantitative method for determining the chemical composition of Periplocaforrestii Schltr. and other ethnomedicines.

As Periplocaforrestii Schltr. is a Chinese herbal medicine with immunosuppressive activity, oleanolic acid is an important quality control component (Yang et al., 1997; Liu et al., 2008; Huang et al., 2010). In previous works, we observed a positive correlation between the oleanolic acid content and the total saponin content of Periplocaforrestii Schltr. The total saponin content can be determined by photometric methods. As spectrophotometers are low-cost instruments, photometric methods can be applied more easily than chromatographic methods to facilitate drug identification in ethnic areas. As a result, the establishment of a model that predicts the content of oleanolic acid in Periplocaforrestii Schltr. from the total content of saponin would allow extensive local detection of this species. 


\section{MATERIALS AND METHODS}

Materials: Ursolic acid (batch number: MUST13020602) and oleanolic acid (batch number: MUST13041606) standards were obtained from the China National Institute for the Control of Pharmaceutical and Biological Products. Ethanol, petroleum ether, $n$-butanol, methanol, vanillin, glacial acetic acid, perchloric acid, and ammonium acetate were analytically pure, and methanol was chromatographically pure. The Periplocaforrestii Schltr. samples used in this study were qualified by Professor Wei Shenghua from the Department of Appraisal of Guiyang College of Traditional Chinese Medicine.

Instrumentation: HPLC measurements were performed using an LC-20AT high-performance liquid chromatograph (Shimadzu Co.) equipped with a Welchrom C18 column $(250 \mathrm{~mm} \times 4.6 \mathrm{~mm}, 5$ $\mu \mathrm{m})$. UV2501PC Ultraviolet-Visible Spectrophotometer (Shimadzu Co.) was used for absorption measurements. Acquisition and processing of the chromatographic data was performed using the Shimadzu LCSoution Lite Chem workstation. Other instruments used in this study included an FW135 Chinese herbal medicine grinder (Tianjin Taisite Instrument Co., Ltd.), a DK-98-1 electric thermostatic water bath (Tianjin Taisite Instrument Co., Ltd.), a GZX-GF101-1-S-II electric thermostatic air-dry oven (Shanghai Hede Experimental Instrument Co., Ltd.), a KQ5200 ultrasonic cleaner (Kunshan Ultrasonic Instrument Co., Ltd.), and a JT1003 precision electronic balance (YuyaoJinnuo Balance Instrument Co., Ltd.).

Spectrophotometric determination of the total saponin content in Periplocaforrestii Schltr: A standard solution of the reference compound ursolic acid was prepared by accurately weighing ursolic acid $(5.030 \mathrm{mg})$ into a $5 \mathrm{~mL}$ volumetric flask. After diluting to volume with methanol, the solution was shaken to obtain the standard solution.

A test solution was prepared by accurately weighing dried Periplocaforrestii Schltr. powder $(2.0 \mathrm{~g})$ into a round-bottom flask. After adding $80 \mathrm{~mL}$ of $70 \%$ ethanol, the solution was placed in an $80^{\circ} \mathrm{C}$ water bath for $3 \mathrm{~h}$, and then cooled and filtered. The combined filtrate was subsequently concentrated to a dry powder, which was redissolved in $25 \mathrm{~mL}$ of water. Then, the solution was extracted three times with $25 \mathrm{~mL}$ of petroleum ether. After removing the petroleum ether layer, the water layer was further extracted three times with $25 \mathrm{~mL}$ of $n$-butanol saturated with water. The solvent was evaporated from the combined $n$-butanol solution in a water bath. The test solution was then prepared by diluting to volume with methanol in a $10 \mathrm{~mL}$ volumetric flask.
To determine the maximum absorption wavelengths of the standard and test solutions, 1 mLof each solution was aspirated into a sealed test tube. After the solvent was evaporated in a water bath, $0.2 \mathrm{~mL}$ of $5 \%$ vanillin-glacial acetic acid solution and $0.8 \mathrm{~mL}$ of $70 \%$ perchloric acid solution were added and the solution was heated for $15 \mathrm{~min}$ in a $60{ }^{\circ} \mathrm{C}$ water bath. Once the test tube was removed from the water bath, it was immediately cooled in ice water for $5 \mathrm{~min}$, and then made up to a volume of $25 \mathrm{~mL}$ with glacial acetic acid. Subsequently, $0.5 \mathrm{~mL}$ of this solution was added to $5 \mathrm{~mL}$ of glacial acetic acid and the mixture was shaken well. A blank reference was prepared through the same method by using methanol instead of the sample solution. The absorbance values were measured in the range of 200 $700 \mathrm{~nm}$. Both the test and standard compounds exhibited a maximum absorption wavelength at $546 \mathrm{~nm}$.

To prepare a standard curve, or plot, $2.5 \mathrm{~mL}$ of the standard solution was aspirated into a test tube and then diluted to $5 \mathrm{~mL}$. Subsequently, 0, 0.2, 0.4, 0.6, 0.8, 1.0 , and $1.2 \mathrm{~mL}$ aliquots of the standard solution were aspirated into six sealed test tubes. These solutions were subsequently reacted with $0.2 \mathrm{~mL}$ of $5 \%$ vanillin-glacial acetic acid solution and $0.8 \mathrm{~mL}$ of $70 \%$ perchloric acid solution and were treated following the same procedure discussed above. The absorbance of each sample was measured at a wavelength of $546 \mathrm{~nm}$ using the solution in the first test tube as the blank control. Finally, the standard curve was constructed with the absorbance as the ordinate. The linear regression equation of the standard curve was $\mathrm{y}=10.699 \mathrm{x}+0.1028(\mathrm{r}=0.9991)$. The two variables showed a good linear relationship within the concentration range of 0.01006-0.06036 $\mathrm{mg} / \mathrm{mL}$ ursolic acid.

Validation of the spectrophotometric method: The precision of this method was evaluated by preparing a test solution using $2.0 \mathrm{~g}$ of Periplocaforrestii Schltr.), as described above. After color development, the absorbance at $546 \mathrm{~nm}$ was measured five times. The RSD value of these measurements was $0.52 \%(n=5)$, indicating that the spectrophotometric method demonstrated good precision.

The stability of the above test solution was investigated by measuring the absorbance every $5 \mathrm{~min}$ over $25 \mathrm{~min}$. The RSD value of these measurements was $0.88 \%(n=6)$, indicating that the absorbance did not change significantly over $25 \mathrm{~min}$. Thus, the test solution following color development was stable over $25 \mathrm{~min}$.

To evaluate the reproducibility, six test solutions were prepared, each with $2.0 \mathrm{~g}$ of Periplocaforrestii Schltr., as described above. After color development, the absorbance of each test solution was then measured at $546 \mathrm{~nm}$. An average content of $0.487 \%$ was determined, with an RSD value of $2.6 \%$, indicating the good reproducibility of the method. 
Sample recovery was investigated by weighing out six 2.0 g portions of the sample (Periplocaforrestii Schltr.) and adding equal amounts of the reference (ursolic acid). The solutions were prepared and color was developed according to the aforementioned method. Subsequently, the absorbance of each sample was measured at $546 \mathrm{~nm}$. The average recovery rate was calculated as $98.85 \%$, with an RSD value of $2.26 \%$, indicating that the method is feasible.

The total saponin content in real samples was determined by weighing out appropriate amounts of different batches of Periplocaforrestii Schltr. powder and preparing test solutions using the above-described method. Using a blank reagent solution as the reference, the absorbance of each sample was measured at $546 \mathrm{~nm}$. The total saponin contents of the samples were then calculated using the regression equation of the standard curve. The results are summarized in Table 1.

HPLC determination of the oleanolic acid content in Periplocaforrestii Schltr: The chromatographic conditions were as follows: chromatographic column, Welchrom C18; mobile phase, methanol-0.1 mol/L ammonium acetate (83:17); flow rate, $1.0 \mathrm{~mL} / \mathrm{min}$; column temperature, $35^{\circ} \mathrm{C}$; detection wavelength, 210 $\mathrm{nm}$; and injection volume, $10 \mu \mathrm{L}$.

A reference solution was prepared by accurately weighing oleanolic acid (4.973 mg) into a $5 \mathrm{~mL}$ volumetric flask. After diluting to volume with methanol, the solution was shaken to obtain the reference stock solution.

To prepare the test solution, $3 \mathrm{~g}$ of dried Periplocaforrestii Schltr. powder was accurately weighed into in a sealed conical flask. After adding $50 \mathrm{~mL}$ of methanol, the solution was ultrasonicated for $1 \mathrm{~h}$, and then shaken, filtered, and evaporated. Subsequently, the residue was redissolved in methanol and transferred to a $25 \mathrm{~mL}$ volumetric flask. After diluting to volume with methanol, the solution was filtered through a $0.45 \mu \mathrm{m}$ microporous membrane. The filtrate was used as the test solution.

Validation of the HPLC method: The linearity of the HPLC method was investigated by aspirating and injecting $2,3,4,6,8$, and $9 \mu \mathrm{L}$ of the reference stock solution into the HPLC system. A standard curve was constructed with the injection volume as the abscissa and the peak area as the ordinate. The linear regression equation of the standard curve was $y=99956 x-46626(r$ $=0.9993)$. The two variables showed a good linear relationship within the range of 1.2-5.4 $\mu \mathrm{g}$ oleanolic acid.

The precision was evaluated by performing five continuous injections of the reference solution $(4 \mu \mathrm{L}$ each). The RSD value of these measurements was $2.7 \%$, indicating that the method had good precision.

The stability of the test solution was evaluated by injecting $10 \mu \mathrm{L}$ of this solution into the HPLC system at $0,6,12,18$, and $24 \mathrm{~h}$. The RSD value for the obtained peak areas was $2.41 \%$. As the peak area did not change significantly, these results indicated that the test solution was generally stable over $32 \mathrm{~h}$.

To evaluate the reproducibility, six test solutions were prepared, each with $3.0 \mathrm{~g}$ of Periplocaforrestii Schltr. from the same batch. These six test solutions were then evaluated under the aforementioned chromatographic conditions. An average content of $0.26 \%$ was determined with an RSD value of $2.1 \%$, which indicated that the method showed good reproducibility.

Sample recovery was investigated by weighing out six $1.5 \mathrm{~g}$ portions of the same batch of Periplocaforrestii Schltr. and adding $1 \mathrm{~mL}$ of the oleanolic acid reference stock solution. The solutions were then prepared according to the above-described method for the test solutions and evaluated under the same chromatographic conditions. The average recovery rate was calculated as $102.61 \%$, with an RSD value of $1.39 \%$, indicating that the method is feasible.

The total oleanolic acid content in real samples was determined by weighing out appropriate amounts of different batches of Periplocaforrestii Schltr. and preparing test solutions using the above-described method listed. The solution was then evaluated under the same chromatographic conditions, and the content of oleanolic acid in the sample was calculated using the regression equation of the standard curve. The results are summarized in Table 1.

\section{RESULTS AND DISCUSSION}

Total saponin content in Periplocaforrestii Schltr: To establish a prediction model for the content of oleanolic acid in Periplocaforrestii Schltr., we first determined the total saponin content using a spectrophotometric method. The saponin contents of the prepared Periplocaforrestii Schltr. test solutions were determined after color development by reacting with $5 \%$ vanillin and perchloric acid. Using the absorbance of the test solution at $546 \mathrm{~nm}$, the content of total saponins in Periplocaforrestii Schltr. was determined based on the standard curve of ursolic acid. The linearity of the standard curve was satisfactory in the range of $0.01006-0.06036 \mathrm{mg} / \mathrm{mL}$ ursolic acid. The test solutions used in this method showed good stability over at least 25 min. Further, this method showed good precision, reproducibility, and recovery. The average recovery was $98.85 \%$ with a relative standard deviation (RSD) of $2.26 \%$.

Oleanolic acid content in Periplocaforrestii Schltr: Subsequently, the content of oleanolic acid in Periplocaforrestii Schltr. was determined using an HPLC method. Gradient elution with a mobile phase of methanol-0.1 mol/L ammonium acetate (83:17) was 
used. Under the tested chromatographic conditions, the retention time of oleanolic acid was $20.055 \mathrm{~min}$ and the peak shape was good. Oleanolic acid was detected at 210 $\mathrm{nm}$ and the constructed standard curve showed satisfactory linearity in the range of $1.2-5.4 \mu \mathrm{g}(\mathrm{r}=$ $0.9993, \mathrm{n}=6$ ). The test solution showed good stability over 32 h. Further, this method showed good precision, reproducibility, and recovery. The average recovery was $102.61 \%$ with an RSD of $1.39 \%$.
Prediction model for the content of oleanolic acid: To avoid restricting the application scope of the prediction model as a result of a close genetic relationship between medicinal materials, five groups of Periplocaforrestii Schltr. samples with different places of origin were selected. The total saponin content and the oleanolic acid content were determined using the above-described methods and the values obtained for each Periplocaforrestii Schltr. sample are shown in Table 1.

Table 1. Total saponin and oleanolic acid contents in Periplocaforrestii Schltr. samples.

Sample no.
S1
S2
S3
S4

S5

\author{
Place of origin \\ Guizhou, Wudang District, Guodihui \\ Guizhou, Guijiaqiao \\ Guizhou, Xixiu District, Sanhe Village \\ Guizhou, Zhijin County, Sanjia \\ Panjiazhai \\ Guizhou, Xiuwen, Xiaojing \\ Pearson correlation coefficien
}

Total saponin, $\%$

0.25

0.49

0.52

Township,

0.75

0.78

0.99

Oleanolic acid $^{\#, ~ \%}$
0.05
0.26
0.27
0.53
0.62

\#The oleanolic acid contents shown in this table were determined using the HPLC method.
As shown in Table 1, the contents of both saponin and oleanolic acid vary significantly in Periplocaforrestii Schltr. samples of different origins, which facilitates the establishment of a generalized prediction model for oleanolic acid. In addition, although variations in the two contents are observed between different medicinal materials, their Pearson correlation coefficient is 0.99 , indicating an obvious positive correlation. This result suggests that it is reasonable to use these two indicators to establish a prediction model.
As the correlation between the total saponin content and the oleanolic acid content in Periplocaforrestii Schltr. demonstrated a significant polynomial trend, a parabolic function was adopted to establish the prediction model for oleanolic acid in Periplocaforrestii Schltr., as shown in Fig. 1. The parabolic fit exhibited a fitting degree $\left(\mathrm{R}^{2}\right.$ value) of 0.987 , suggesting that this model reproduces the observed correlation between the total saponin content and the oleanolic acid content in Periplocaforrestii Schltr.

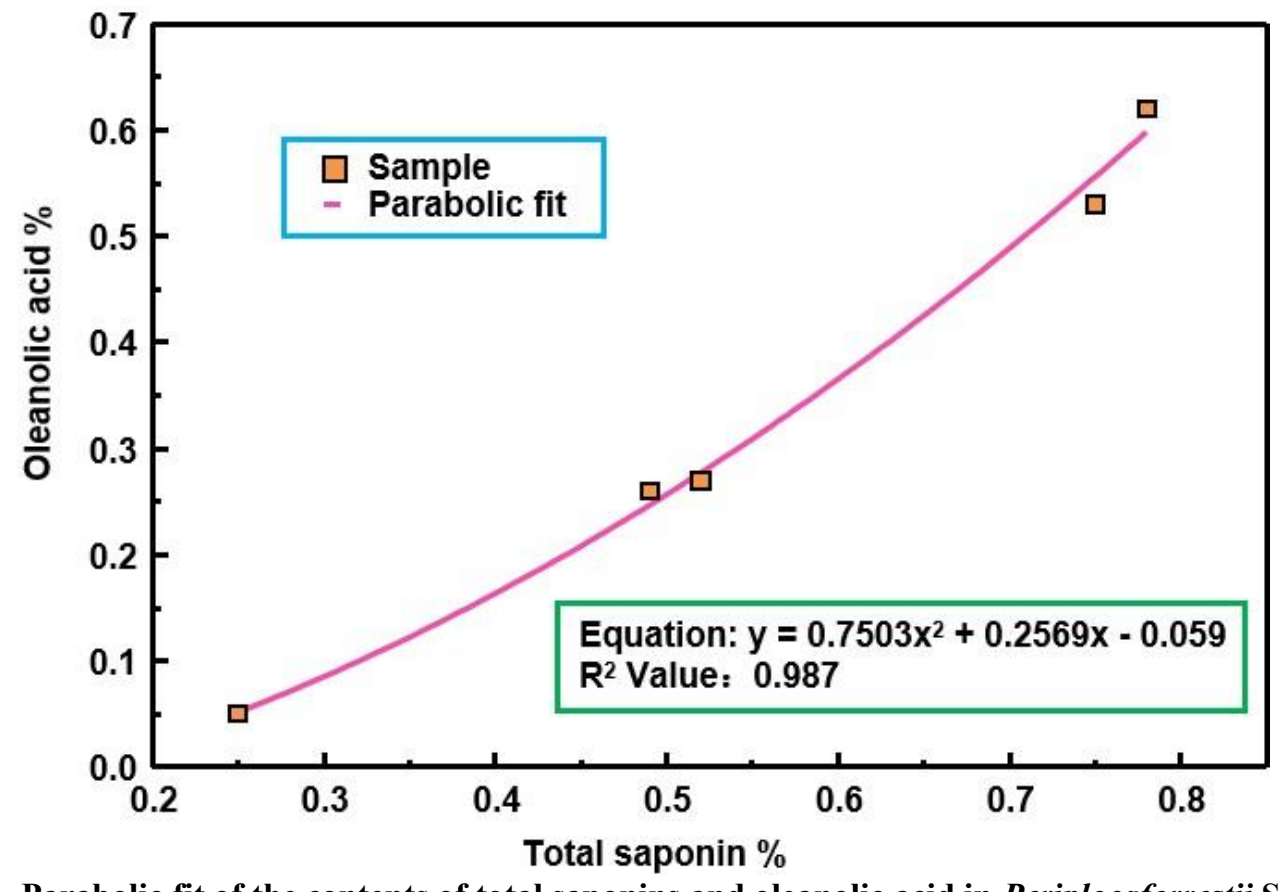

Fig. 1. Parabolic fit of the contents of total saponins and oleanolic acid in Periplocaforrestii Schltr. 
To validate the above model, the oleanolic acid contents of two groups of Periplocaforrestii Schltr. were predicted and compared to the HPLC results (Table 2). For the two groups of Periplocaforrestii Schltr., the difference between the value predicted by the model established in this study and the actual value determined by HPLC analysis is less than $3 \%$, indicating that the model is reasonably accurate within the oleanolic acid content range of $0.05-0.67$.

Table 2. Predicted and experimentally determined contents of oleanolic acid in Periplocaforrestii Schltr.

\begin{tabular}{llll}
\hline Sample no. & $\begin{array}{l}\text { Total } \\
\text { saponin, } \%\end{array}$ & \multicolumn{2}{l}{ Oleanolic acid, \% } \\
\cline { 3 - 4 } & $\begin{array}{l}\text { Predicted } \\
\text { value }\end{array}$ & $\begin{array}{l}\text { Detected } \\
\text { value }\end{array}$ \\
\hline $\mathrm{T} 1$ & 0.84 & 0.69 & 0.67 \\
$\mathrm{~T} 2$ & 0.60 & 0.37 & 0.36 \\
\hline
\end{tabular}

In order to reduce the costs of chemical content detection for plants, large number of studies have been carried out by phytochemists. Even so, it was still difficult to detect active components of natural drugs by using these methods. Because most of them were just suitable for the total content detection of a class of compounds, not for specific single one (Buysse et al., 1993). But the active component of natural medicine is usually a single compound, such as oleanolic acid which is an important active component of Chinese herbal medicines with immunosuppressive activity. Until now, the determination of the content of oleanolic acid mainly relies on various high-precision chromatographic and mass spectrometric detection methods which are a heavy burden for economically underdeveloped ethnic areas.

Establishing a component prediction model is a fast, accurate and economical methods for analysis of the component. But this method is mainly used in the field of agriculture (Bagchi et al., 2016). It has hardly been used in the analysis of natural pharmaceutical ingredients.

In previous works, we had observed a positive correlation between the oleanolic acid content and the total saponin content of Periplocaforrestii Schltr. The establishment of a model that predicts the content of oleanolic acid in Periplocaforrestii Schltr. from the total content of saponin which can be determined by low-cost and rapid photometric methods, would allow extensive local detection of this species. But, in-depth research is still needed for the practical application of this model.

Conclusion: In this study, a significant positive correlation between the total saponin content and oleanolic acid in Periplocaforrestii Schltr. within a certain range was confirmed by testing Periplocaforrestii Schltr. samples from five different origins. The high Pearson correlation coefficient between these indicators
(0.99) indicated the feasibility of establishing a prediction model, and the parabolic fitting curve for these two indicators showed a good degree of fitting (0.987). Application of the developed model to predicting the oleanolic acid contents in two groups of Periplocaforrestii Schltr. samples produced values within $3 \%$ of the contents determined by the HPLC detection method, suggesting that the model was accurate within a certain range.

As the detection of total saponin can be performed using just a low-cost spectrophotometer and the operational steps of this method are much simpler than those of high-precision detection methods, the established prediction model provides a possible method for rapid identification of natural medicinal materials in ethnic areas.

Acknowledgments: This work was financially supported by National Natural Science Foundation of China (No: 81660710).

\section{REFERENCES}

Bagchi, T.B., S. Sharma, and K. Chattopadhyay (2016). Development of NIRS models to predict protein and amylose content of brown rice and proximate compositions of rice bran. F. Chem. 191, 21-27.

Buysse, J., and R. Merckx (1993). An Improved Colorimetric Method to Quantify Sugar Content of Plant Tissue. J. Exper. Bota. 44, 1627-1629.

Chen, L., J. Li, X.Ke, W. Qu, J. Zhang, F. Feng and W. Liu (2017). The therapeutic effects of Periplocaforrestii Schltr. Stem extracts on collagen-induced arthritis by inhibiting the activation of Src/NF- $\mathrm{kB}$ signaling pathway in rats. J.Ethnopharmacol. 202, 12-19.

Chen, Q., F.Y.Jin, G.H. Chen andL.Y.Yang (2005). Study on Screening of Effective Parts of Periplocaforrestii Schltr. Chin. J.Ethnomed.Ethnopharm. 74, 163-165.

Hu, X.N., X. Zhou, M. Li, H.Liu,and W.Zhou (2007).Analysis on Chemical Constituents of Volatile Oil of the Fruit of Periplocaforrestii Schltr. J Instrum Anal. 26, 160-163.

Huang, Z.F., Z.H. Huang, J.L.Hu , Y.H.Zhou and D.L. Wei (2010). Extracting and Identification of OA and ABPS of local achyranthes aspera.China Pract. Med. 5, 6-7.

Zhang, L.L., Z.Y. Xiao, Y. Ma, C.H. Qi, W.X. Zhou, Y.X. Zhang, S.Y.Qiao and L. Sun (2011).Cellular immunosuppression effect of polysaccharides HGT-5A from Periplocaforrestii Schlecht and its possible active components. Chin. J.Pharmacol.Toxicol. 25, 538-542.

Li, Q., Y. Chen, Q.Y. Zhou, X.Li, D.B. Yuan and H. Zhang (2015). Simultaneous Determination of 
Three Components in the Extract of Compound Radix Caulis Sarcandrae by High Performance Liquid Chromatograph. J. Chin. Med. Mater. 38, 2184-2186.

Liang, J., W.K. Ma, Z.Q. Liu, Y.An, X.M. Yao and Z.Y. Tang (2015). Effects of Periplocaforrestii Schltr. ethanol extract (PFE) on proliferation, COX-2 expression, and PG2 expression of Rheumatoid arthritis synovial fibroblasts from patients. Lishizhen Med. Mater. Med. Res. 26, 24-27.

Liang, Q., H. Chen, X. Zhou, Q.Deng, E. Hu, C. Zhao and X. Gong (2017).Optimized microwaveassistant extraction combined ultrasonic pretreatment of flavonoids from PeriplocaforrestiirSchltr. and evaluation of its antiallergic activity. Electrophoresis 38, 1113-1121.

Liu, S.H., J.Q. Wang, Y. Liu and J.L. Jia (2008). Determination of oleanolic acid in mistletoe by high performance liquid chromatography. Chin.Tradit. Pat. Med. 30, 926-928.

Lu, M.S., H. Zhang, G. Liu, X.Y. Zhang, C.P.Huang (2011).Effect of Periplocaforrestii Schltr. compound extracts on TNF- $\alpha$, MMP-9 and TIMP-1 in synovial tissues of rats with adjuvant arthritis. Lishizhen Med. Mater. Med. Res. 22, 2593.

$\mathrm{Xu}$, R., Y.H. Zhang and Y.T. Zhao (2011).Study on Chemical Constituents of Periplocaforrestii Schlecht. Chin. Pharm. J. 11, 823-826.

Xu, Y., Q. Li, J. Yao, H.M.Li, X.F. Zhang and H.Zhang (2016). Simultaneous determination of nine effective ingredients in compound Heiguteng by HPLC. Chin. J. Pharm. Anal. 36, 1190-1198.

Yang, J.F. and L.J.Shen (1997).Determination of Hesperidin and Oleanolic Acid in Wujiapi Wine by HPLC. Chin.Tradit. Pat. Med. 19, 8-9.

Zhao, C., X.H. Gan, X.J. Gong, and H.G. Chen and X. Zhou (2014).Ceramides of Periplocaforrestii. Schlecht. Chin. J. Exp.Tradit. Med. Formulae. 23, 83-85.

Zhao, X.L., H. Zhang, X.Y. Li, H.M. Li, Q.Y. Zhou and Q. Li (2016).Studies on ultra performance liquid chromatography characteristic chromatogram of Periplocaforrestil Schltr. Chin. J. Pharm. Anal. 36, 1963-1970. 